\title{
FREQÜÊNCIA DE EFEITOS ADVERSOS CAUSADOS POR INIBIDORES DA ENZIMA CONVERSORA DA ANGIOTENSINA RELATADOS POR PACIENTES ATENDIDOS NA UNIDADE BÁSICA DE SAÚDE - LARANJAL - PR
}

\author{
FREQUENCY OF ADVERSE EFFECTS CAUSED BY CONVERTING ENZYME \\ INHIBITORS ANGIOTENSIN RELATEDES BY PATIENTS ASSISTED AT THE UNIT \\ OF BASIC HEALTH - LARANJAL - PR
}

\author{
BARBOSA, A. A. M.1; DUDEK, D. N. ${ }^{2}$; MARIA, M. D.3; SANCHES, A. C. C. ${ }^{4}$ \\ ${ }^{1}$ Aluna do Curso de Pós-Graduação Lato Sensu em Farmacologia Aplicada da Universidade Estadual do Oeste do Paraná, \\ Unioeste, Cascavel, PR, Brasil. ${ }^{2,3}$ Acadêmicas do Curso de Farmácia da Unioeste. ${ }^{4}$ Coordenadora do Curso de Pós- \\ Graduação Lato Sensu em Farmacologia Aplicada, Unioeste. \\ RECEBIDO: 012/06/08 ACEITO:30/06/08
}

\section{RESUMO:}

Os inibidores da enzima conversora da angiotensina (ECA) têm alta eficácia no tratamento da pressão arterial elevada, bem como a ser utilizado em situações de insuficiência cardíaca. $O$ mecanismo de ação desses medicamentos é fornecido pela inibição da formação de angiotensina II, um potente agente vasoconstritor, através do bloqueio da ECA. Os efeitos adversos mais comuns são: tosse, hipotensão ortostática na primeira dose, erupção cutânea, perda do paladar, proteinúria, leucopenia e hipersensibilidade com edema angioneurótico. O estudo realizado é a avaliação do efeito colateral dos inibidores da enzima conversora da angiotensina. Os resultados foram analisados através do programa Epi Info 3.3.2 de 9 de fevereiro de 2005. Entre os itens variáveis sócio-demográficas, o sexo feminino tem $71,7 \%$ da amostra, a média de idade de 53 anos e ensino fundamental incompleto $67,9 \%$ das pessoas entrevistadas. Em relação ao estado civil, $83,0 \%$ eram casados, quanto ao regime domiciliar $51,9 \%$ pertenciam à primeira geração (com um cônjuge), sendo 66,0\% tem como renda mensal 1 (um) salário mínimo. Em relação as variáveis farmacoepidemiológicas alguns dos resultados encontrados foram, o medicamento de uso mais freqüente foi o inibidor da ECA (Captopril), prescrito para $67,9 \%$ dos hipertensos, com aparecimento de tosse em aproximadamente 3 a $20 \%$ dos pacientes. Com relação à adesão ao tratamento $86,8 \%$ respondem positivamente. Diante da realidade assinalada é necessário aumentar o grau de conhecimento da população sobre a importância do controle da hipertensão arterial, e a adesão à terapêutica cardiovascular. É necessário e urgente garantir acesso dos pacientes hipertensos a serviços básicos de saúde, e incentivar políticas e programas comunitários. O primeiro passo é a educação dos pacientes, seus familiares e a comunidade, onde uma equipe multiprofissional poderá fornecer aos pacientes e à comunidade motivação para vencer os desafios e adotar atitudes que tornem as ações efetivas e permanentes.

Palavras-chave: Inibidores da enzima conversora da angiotensina, tosse, pressão arterial alta.

\section{ABSTRACT:}

The inhibitors of the converting enzyme of the angiotensin (ECA) have high effectiveness in the treatment of the high blood pressure, as well as being used in situations of heart failure. The mechanism of action of these drugs is provided by the inhibition of the formation of angiotensin II, a powerful vasoconstrictor agent, through the blockade of the ECA. The more common adverse effects are: cough, orthostatic hypotension of first dose, cutaneous eruption, loss of the palate, proteinuria, leucopenia and hypersensibility with angioneurotic edema. The study performed is a avaliated the collateral effect of inhibitors of the converting enzyme of the angiotensin. The results had been analyzed through the Epi Info 3.3.2 program of February 9th, 2005. Amongst the socialdemographic variable items, the feminine sex got $71.7 \%$ of the sample, average age of 53 years and incomplete basic education $67.9 \%$ of the interviewed people. In relation to civil state, $83,0 \%$ were married, while to domiciliary arrangements $51.9 \%$ belonged to the first generation (with a spouse), being $66.0 \%$ on 1 minimum wage level.For some of the variables 
Pharmacoepidemiological results were the most frequent drug use was the ACE inhibitor (captopril), prescribed for $67.9 \%$ of hypertensive patients, with the emergence of approximately 3 to $20 \%$ of patients. With regard to adherence to treatment $86.8 \%$ answered positively. Facing the reality is marked to increase the awareness of population about the importance of control of hypertension, and adherence to cardiovascular therapy. It is an urgent need to ensure access of hypertensive patients to basic health services, and encourage policies and programs. The first step is education of patients, their families and the community where a team can provide to patients and community motivation to overcome the challenges and adopt attitudes that make actions effective and permanent.

Keywords: Inhibitors of the converting enzyme of the angiotensin, Cough, High blood pressure.

\section{INTRODUÇÃO}

A hipertensão arterial constitui-se numa das afecções mais comuns do mundo moderno e atinge em média, de $15 \%$ a $20 \%$ da população adulta (SIMONETTI, BATISTA e CARVALHO 2002).

De acordo com V Diretrizes Brasileiras de Hipertensão Arterial (2006) preconiza que o diagnóstico seja realizado o mais precocemente possível, além do tratamento adequado e contínuo, é uma arma importante no tratamento da hipertensão; entretanto, a prevenção do aumento da pressão arterial constitui o meio mais eficiente de combatê-la, evitando as dificuldades e o elevado custo social de seu tratamento e de suas complicações.

O tratamento medicamentoso deve ser individualizado e ter como princípios gerais a eficácia por via oral e boa tolerância, além de permitir a administração do menor número possível de tomadas diárias, com preferência para aqueles com posologia de dose única diária ( RAMOS, 1995).

Deve-se ainda iniciar com as menores doses efetivas preconizadas para cada situação clínica, podendo ser aumentadas gradativamente e/ou associadas a outro hipotensor de classe farmacológica diferente (deve-se levar em conta que quanto maior a dose, maiores serão as probabilidades de efeitos indesejáveis); respeitar um período mínimo de quatro semanas para se proceder ao aumento da dose e ou a associação de drogas, salvo em situações especiais; instruir o paciente sobre a doença, efeitos colaterais dos medicamentos, planificação e objetivos terapêuticos; considerar as condições socioeconômicas (I DIRETRIZ, 2002).

Os inibidores da ECA estão hoje entre os anti-hipertensivos com o maior número de indicações terapêuticas, além da hipertensão arterial. São hipotensores muito eficazes, tanto em monoterapia como em associação com outras drogas, especialmente diurético (IV DIRETRIZES, 2002).

O emprego do IECA tem sua maior indicação no idoso hipertenso com associação de insuficiência cardíaca e/ou diabetes (BECKTE, FLETCHER, BULPITT, 2001). Como monoterapia reduzem a pressão arterial em mais de $50 \%$ dos pacientes com hipertensão arterial leve e moderada. Em geral, brancos hipertensos respondem melhor do que negros; jovens melhor do que idosos (SILVA, 2006).

O Captopril foi o primeiro IECA descoberto e ainda é considerado o medicamento padrão ouro nesta classe de drogas anti-hipertensivas (BIONDO-SIMÕES, 2006. A tosse é o principal e mais freqüente efeito colateral promovido pelos inibidores da ECA. Em pacientes desidratados ou com tratamento diurético intenso pode ocorrer hipotensão. Outra condição onde os inibidores da ECA são contra-indicados é a gravidez, pois estes podem levar à lesão fetal e morte. Outros efeitos colaterais bem menos freqüentes incluem: edema angioneurótico, erupção cutânea, urticária e perda do paladar (WHO, 2004). 
De acordo com LORGA et al (2002), os inibidores da enzima de conversão podem ser utilizados em idosos para tratamento da hipertensão arterial. Embora sem evidências clínicas diretas de efeito sobre a mortalidade em idosos hipertensos, mostraram-se tão eficazes quanto os diuréticos e betabloqueadores na redução da mortalidade cardiovascular. O objetivo do tratamento da hipertensão é reduzir o risco de morbidade e de mortalidade devida às lesões provocadas em órgãos-alvo. O uso de inibidores da enzima conversora de angiotensina (IECA) é uma importante opção terapêutica (BIONDO-SIMOES, 2006).

\section{MATERIAL E MÉTODOS}

Este estudo caracterizou-se como sendo do tipo descritivo, de caráter educativo, no período de 15 de março a 17 de maio de 2007, na Unidade Básica de Saúde de Laranjal Paraná, que está integrado ao programa Hiperdia do Ministério da Saúde, na qual foram feitas palestras e entrevistados 45 idosos hipertenso e 8 idosos hipertenso/diabéticos que utilizavam inibidor da enzima conversora de angiotensina (Captopril). Este estudo foi desenvolvido a partir da elaboração de um formulário semi-estruturado composto por perguntas simples, diretas e de fácil compreensão, contendo três partes: a primeira relacionada às variáveis sóciodemográficas (idade, sexo, estado civil, renda, escolaridade), a segunda variável farmacoepidemiológica (medicamentos prescritos, concentração, horário, forma de administração, interação medicamento/alimento, efeitos adversos), e a terceira parte medidas educativas (reforço para: dose, horário, interação, efeito adverso, armazenamento, forma correta de administração, dieta e exercícios físicos). Os objetivos, a importância da pesquisa e termo de consentimento livre e esclarecido foi apresentada aos pacientes concordantes, sendo que não houve nenhuma recusa em participar da pesquisa. As palestras foram elaboradas, utilizando-se de conteúdos de livros, visando explicar, exemplificar e esclarecer dúvidas sobre a hipertensão arterial, como também os fatores de risco, reações adversas dos medicamentos, consumo de sal, estar constantemente aferição da pressão arterial e a importância de adesão ao tratamento. Para a coleta de dados e análise foi utilizado o programa Epi Info 3.3.2, Versão Windows de 09 de fevereiro de 2005.

\section{RESULTADOS E DISCUSSÃO}

Entre os 53 indivíduos estudados, foram observados que $84,90 \%$ eram hipertensos e $11,30 \%$ hipertenso/diabético (fig. 1).

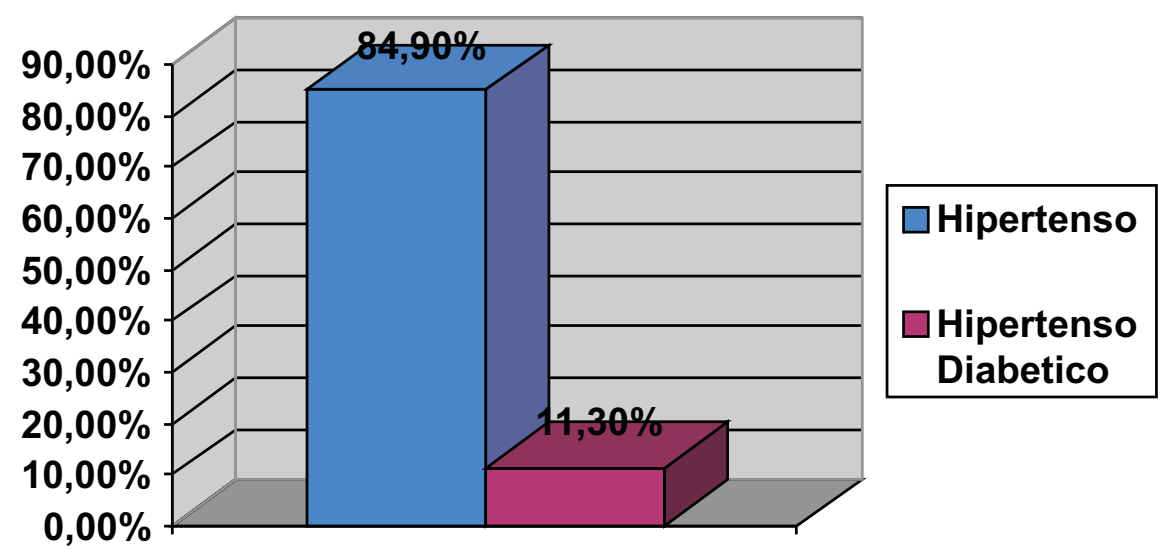

FIGURA 1. Percentual de idosos hipertensos e idosos hipertenso/diabéticos que participaram do estudo na Unidade Básica de Saúde de Laranjal - Pr 
Em relação às variáveis sócio-demográficas (tabela. 1) a maior freqüência foi para o sexo feminino (71,7\%), idade média foi de 53 anos, com extensão de 38 a 68 , e estado civil (casados) $83,0 \%$. Estudos realizado por BERTOLDI et al (2004), mostra que as mulheres possuem maior preocupação com a saúde e procuram mais os serviços de saúde do que os homens. Em relação à escolaridade, o ensino fundamental incompleto foi o mais freqüente $67,9 \%$. SANS et al (2002) relata que os indivíduos com 60 anos ou mais apresentam maior proporção de ausência de escolaridade ( $23 \%$ comparado com $3,5 \%$ nos outros grupos etários).

$\mathrm{O}$ arranjo familiar (com quem o paciente reside) mostra que 52,8\% pertencem à primeira geração (reside apenas com o cônjuge), cabe ressaltar que, na maioria das vezes, o cônjuge é idoso ou muito próximo dessa faixa etária e seu potencial de auxílio pode estar limitado. Outros estudos vêm mostrando resultados diferentes do encontrado na atual pesquisa. LEBRAO e LAURENTI (2005) descrevem que os idosos co-residem com cônjuge e/ou filhos (sem netos) em primeiro lugar; em segundo lugar, somente com cônjuges; e em terceiro, em arranjo trigeracional, em quarto, sozinhos.

No que diz respeito à renda familiar constatou-se nível relativamente baixo, $66,0 \%$ dos entrevistados recebiam um salário mínimo. Aspecto que chama a atenção é o fato de que grande parte dos idosos apresenta rendimento insuficiente para uma vida digna, Segundo CAMARANO et al (1999), a educação e rendimento estão fortemente associados a melhores condições de vida.

Tabela 1: Variável sócio-demográfica de pacientes hipertensos da Unidade Básica de Laranjal - Pr, 15 de março a 17 de maio de 2007.

\begin{tabular}{llllll}
\hline Sexo & Número & $\%$ & Estado Civil & Número & $\%$ \\
Masculino & 15 & $28,3 \%$ & Casado & 44 & $83,0 \%$ \\
Feminino & 38 & $71,7 \%$ & Divorciado & 02 & $3,8 \%$ \\
Total & 53 & $100 \%$ & Solteiro & 01 & $1,9 \%$ \\
& & & Viúvo & 06 & $11,3 \%$ \\
& & & Total & 53 & $100 \%$ \\
Idade(anos) & Número & & Renda (SM) & Número & $\%$ \\
Média & 53 & & 1 SM & 35 & $66,0 \%$ \\
Amplitude & $38-68$ & & 2 a 3 SM & 18 & $34,0 \%$ \\
& & & Total & 53 & $100 \%$ \\
& & & & & \\
Escolaridade & Número & $\%$ & Arranjo & Número & $\%$ \\
& & & Familiar & & \\
Analfabeto & 11 & $20,8 \%$ & Mora só & 03 & $5,7 \%$ \\
Fund/incompleto & 36 & $67,9 \%$ & $1^{a}$ geração & 28 & $52,8 \%$ \\
Fund/completo & 06 & $11,3 \%$ & $2^{\text {a geração }}$ & 22 & $41,5 \%$ \\
Total & 53 & $100 \%$ & & 53 & $100 \%$ \\
\hline
\end{tabular}

Em relação as variáveis farmacoepidemiológicas (tabela 2) o medicamento de uso mais freqüente foi o inibidor da ECA (Captopril), prescrito para $67,9 \%$ dos hipertensos. BIONDO E SIMÕES (2006) relatam que o objetivo da hipertensão é reduzir o risco de morbidade e de 
mortalidade devida às lesões em órgãos - alvo que este distúrbio pode levar. O uso de inibidor da enzima conversora de angiotensina (IECA) é uma importante ferramenta terapêutica.

Tabela 2: Medicamentos prescritos para sistema cardiovascular a pacientes hipertensos atendidos na Unidade Básica de Saúde de Laranjal - Pr, de 15 de março a 17 de maio, 2007.

\begin{tabular}{|c|c|c|c|c|}
\hline $\begin{array}{l}\text { Sistema Anatômico } \\
\text { Terapêutico } \\
\text { Químico }\end{array}$ & $\begin{array}{l}\text { Classe } \\
\text { Terapêutica }\end{array}$ & $\begin{array}{l}\text { Princípio } \\
\text { Ativo }\end{array}$ & Freqüência & $\%$ \\
\hline \multirow{3}{*}{$\begin{array}{l}\text { Sistema } \\
\text { Cardiovascular }\end{array}$} & Inibidor da Eca* & Captopril & 36 & $67,9 \%$ \\
\hline & $\begin{array}{l}\text { Antagonista } \\
\text { Betaadrenérgicos }\end{array}$ & Propanolol & 14 & $26,4 \%$ \\
\hline & $\begin{array}{l}\text { Inibidor } \\
\text { Sistema } \\
\text { Simpático } \\
\text { Central }\end{array}$ & Metildopa & 03 & $5,7 \%$ \\
\hline Total & & & 53 & $100 \%$ \\
\hline
\end{tabular}

*Inibidor da Eca - Inibidor da Enzima Conversora de Angiotensina.

O perfil de medicamentos prescritos associados é mostrado na (tabela 3 ), sendo que o medicamento com maior freqüência no sistema cardiovascular foi o hidroclorotiazida com $(82,9 \%)$.

Tabela 3: Perfil dos medicamentos prescritos associados* a pacientes hipertensos atendido s na Unidade Básica de Saúde de Laranjal - Pr, 15 de março a 17 de maío de 2007.

\begin{tabular}{|c|c|c|c|}
\hline $\begin{array}{l}\text { Sistema } \\
\text { Terapêutico Químico }\end{array}$ & Princípio Ativo & $\begin{array}{l}\text { Freqüênci } \\
\text { a }\end{array}$ & $\%$ \\
\hline \multirow[t]{4}{*}{ Sistema Cardiovascular } & Hidroclorotiazida & 29 & $82,9 \%$ \\
\hline & Furosemida & 02 & $5,6 \%$ \\
\hline & Nifedipina & 03 & $8,6 \%$ \\
\hline & Digoxina & 01 & $2,9 \%$ \\
\hline Total & & 35 & $100 \%$ \\
\hline \multirow[t]{5}{*}{ Sistema Músculo Esquelético } & AAS & 21 & $58,3 \%$ \\
\hline & Diclofenaco & 10 & $27,8 \%$ \\
\hline & Dipirona & 02 & $5,6 \%$ \\
\hline & Nimesulida & 01 & $2,7 \%$ \\
\hline & Ibuprofeno & 02 & $5,6 \%$ \\
\hline Total & & 36 & $100 \%$ \\
\hline
\end{tabular}


Metabolismo Tato Alimentar

Total

Sistema Respiratório

Total

Sistema Nervoso

Glibenclamida
Metformina
Hidróxido de Alumínio
Dimeticona
Omeprazol

Aminofilina

Diazepam

Cloridrato

Amitriptilina

$\begin{array}{ll}2 & 15,4 \% \\ 3 & 23,1 \% \\ 1 & 7,6 \% \\ 3 & 23,1 \% \\ 4 & 30,8 \% \\ 13 & 100 \%\end{array}$

02

02

$100 \%$

$100 \%$

Total

$2 \quad 40,0 \%$

de $3 \quad 60,0 \%$

* Medicamentos Prescritos associados: são medicamentos prescritos conjuntamente com a terapia hipertensiva.

Estudos feitos por SANTELO e MION (1998) mostraram que a associação de captopril com hidroclorotiazida, independentemente do critério empregado para análise, é eficaz e apresenta boa tolerabilidade, estando indicada como monoterapia em dose única diária para hipertensos leves e moderados. Foi demonstrado também que houve aumento da eficácia com o aumento da dose diária. No sistema músculo esqueléteico $(58,3 \%)$ utilizava ácido acetil salicílico $100 \mathrm{mg}$ uma dose diária. O Primary Prevention Project avaliou o uso de AAS $100 \mathrm{mg}$ ao dia em indivíduos com hipertensão, hipercolesterolemia e diabetes, demonstrando uma significativa freqüência menor de morte e eventos cardiovasculares nos pacientes não diabéticos. No subgrupo dos diabéticos, não houve melhora (SACCO, 2003). Quanto as associações de medicamentos prescritos do aparelho respiratório foi observado que 2 pacientes utilizavam aminofilina, e do sistema nervoso $60 \%$ utilizavam cloridrato de amitripitilina. MIYASAKA (2003) relata que a prescrição de múltiplas medicações é uma realidade que requer atenção e cuidado constante. Esse cuidado inclui a revisão das medicações em uso e o conhecimento extensivo destas, sempre tentando minimizar o número de substâncias utilizadas, monitorando e valorizando efeitos colaterais e tóxicos.

A tabela 4 mostra as freqüências de reações adversas causadas pelo inibidor da enzima conversora da angiotensina (Captopril) sendo este o único desta classe dispensada na UBS de Laranjal - Pr.

Tabela 4: Incidência de reações adversas detectadas em pacientes hipertensos atendidos na Unidade Básica de Saúde Laranjal - Pr, 15 de março a 17 de maio, 2007.

\begin{tabular}{|c|c|c|}
\hline Tipo de Intervenção & Freqüência Absoluta & $\begin{array}{l}\text { Freqüência } \\
\text { Relativa }\end{array}$ \\
\hline $\begin{array}{l}\text { Interações } \\
\text { medicamento/medicamento }\end{array}$ & 06 & $31,6 \%$ \\
\hline Interações medicamento/alimento & 10 & $52,6 \%$ \\
\hline Eventos adversos a medicamentos & 03 & $15,8 \%$ \\
\hline Total & 19 & $100 \%$ \\
\hline
\end{tabular}


A maior freqüência foi para interações medicamento/alimento onde 52,6\% associavam o medicamento próximo à refeição. Pesquisa feita por SUCAR (2000), mostra que o captopril apresenta perfil farmacocinético caracterizado por uma excelente absorção no trato gastrintestinal, que poderá ser reduzida em até $40 \%$ pela presença de alimentos. Quanto ao primeiro membro da classe dos inibidores da ECA (captopril), é importante salientar que os pacientes devem ser orientados a ingeri-lo preferencialmente em jejum, ou manter um intervalo de 1 hora antes ou 2 horas após as refeições NAKAO et al (2003).

Em relação à interação medicamento/ medicamento, 31,6\% associam os analgésicos e antiinflamatórios. Outros estudos vêm mostrando resultados iguais ao encontrado na atual pesquisa. SIMÕES e FARACHE (1998) mostram em estudo feito por consumo de medicamentos, que os analgésicos e antiinflamatórios, foram os que apresentavam maior percentual de uso em idosos. Também foi identificado $15,8 \%$ de casos de reações adversas a medicamento, sendo que dos três pacientes, dois apresentavam tosse após o uso do captopril, sendo necessária à interrupção do tratamento devido à intensidade do sintoma, mudando para outra terapia adequada. Estudos realizados por JACOMELLI et al, (2003) mostra que o aparecimento da tosse pode ocorrer em horas a meses após a sua introdução, possivelmente explicado pelo acúmulo de substância $P$, bradicinina e prostaglandina que estimulam as fibras "C" na mucosa respiratória. O aparecimento de tosse tem sido relatado em aproximadamente 3 a $20 \%$ dos pacientes que utilizam essa medicação, não sendo dose-dependente e podendo ocorrer com qualquer tipo de IECA. A melhora após a suspensão da droga pode demorar até quatro semanas, sendo fator determinante do diagnóstico. Outra condição onde os inibidores da ECA são contra-indicados é a gravidez. O outro caso de reação adversa a medicamento era uma paciente gestante, que estava no quinto mês de gestação. PRYDE, THORPE e LAMONT (1993) relatam que o uso dos inibidores da enzima conversora de angiotensina está associado a efeitos colaterais, como oligoidrâmnio, retardo de crescimento intra-uterino, prematuridade, insuficiência renal do concepto, malformação óssea e morte neonatal. Realizouse uma intervenção sugerindo a mudança ao prescritor, tendo sido acatada a sugestão para outra terapia adequada. MIYASAKA (2003) afirma que, o estudo das interações medicamentosas constitui mais um desafio profissional que está apenas começando. A base para sua identificação e compreensão está no conhecimento de fisiologia, fisiopatologia, mecanismo de ação dos fármacos, mas também de uma atenção voltada à observação clínica do paciente, caracterizando sintomas e sua evolução ao longo do tratamento. Provavelmente, o futuro reserva formas mais claras de prevenção e identificação de efeitos farmacológicos indesejados, contribuindo assim para o sucesso terapêutico.

A tabela 5 mostra que dos 53 pacientes entrevistados que fazem uso de pelo menos uma droga anti-hipertensiva $(98,1 \%)$ utilizavam a concentração indicada, o restante se era para tomar três medicamentos/dia, tomavam dois. Em relação ao horário $96,2 \%$ aderiam ao horário correto.

\begin{tabular}{lll}
$\begin{array}{l}\text { Tabela 5: } \\
\text { diabéticos atendidos na Unidade Básica de Saúde de Laranjal - Pr, } 15 \text { de março a } \\
\text { 17 de Maio, 2007. }\end{array}$ & \begin{tabular}{l} 
Freqüência Relativa \\
\hline Fatores quanto a Adesão
\end{tabular} & $\begin{array}{l}\text { Frência } \\
\text { absoluta }\end{array}$ \\
\hline $\begin{array}{l}\text { Concentração } \\
\text { utilizada/paciente }\end{array}$ & 52 & $98,1 \%$ \\
Horário seguido pelo paciente & 51 & $96,2 \%$ \\
Período/tratamento seguido & 46 & $86,8 \%$ \\
\hline
\end{tabular}


Quanto ao período de tratamento seguido, (86,8\%) respondeu afirmativamente, o restante responderam que em algum momento deixavam de tomar a medicação, alguns fornecendo mais de um motivo sendo estes: "esquecimento", "estar se sentindo bem", "o organismo se acostuma sem ele". Todos os outros motivos dizem a respeito a uma opção pessoal ou falta de cuidado com o tratamento.

Como já foi citado a UBS fornece medicação aos mais necessitados, contudo há situações em que o usuário necessita de um tipo específico de medicamento que não está disponível gratuitamente e/ou às vezes na quantidade necessária para uso mensal. Diante deste fato, muitos deles necessitam comprar, o que às vezes, devido à situação financeira precária de uma grande maioria, não o faz. Sabe-se que os medicamentos da área cardiológica possuem um elevado custo, percebe-se que para alguns casos a interrupção do uso do medicamento implica em uma piora do quadro hipertensivo trazendo prejuízo para saúde física do entrevistado. Fica claro ainda, que o usuário com poder aquisitivo baixo depende realmente dos medicamentos fornecidos gratuitamente pelo SUS. ARAÚJO e GARCIA (2006) mostram que através da participação ativa, o portador de hipertensão compartilha a responsabilidade pelo sucesso da terapêutica farmacológica e não farmacológica, ou seja, cumpre os pontos do regime de tratamento no que diz respeito ao uso correto dos medicamentos; comparece aos encontros agendados e monitora o plano terapêutico e seus resultados. Além disso, realiza modificações no estilo de vida, adotando efetivamente o regime dietético prescrito, praticando regularmente atividades físicas e monitorando o estresse.

\section{CONSIDERAÇÕES FINAIS}

O uso dos inibidores da ECA no tratamento da hipertensão e insuficiência cardíaca tem como efeito comum e persistente a tosse não produtiva. A tosse é um sintoma que, dependendo da intensidade, pode tornar-se um fator importante na degradação da qualidade de vida dos pacientes. $O$ constrangimento, seja pelo ruído causado pela tosse perturbando o sono dos pacientes e seus familiares, seja como elemento de gatilho para micção involuntária, é um elemento limitante no dia a dia dos pacientes.

Diante da realidade assinalada é necessário aumentar o grau de conhecimento da população sobre a importância do controle da hipertensão arterial, e a adesão a terapêutica cardiovascular, que por muitas vezes deixam de tomar o medicamento por motivos de reações adversas, sendo que desconhecem este fator. É necessário e urgente garantir acesso dos pacientes hipertensos a serviços básicos de saúde, e incentivar políticas e programas comunitários. O primeiro passo é a educação em saúde na tentativa de desenvolver e estimular o processo de mudança de hábitos e transformação no modo de viver. Essas ações educativas devem ser desenvolvidas com os pacientes, seus familiares e a comunidade por meio de recursos que vão desde o contato individual até a utilização de fontes de informação coletiva, como folhetos, reuniões, palestras, peças teatrais e vídeos.

Como a hipertensão é uma doença multifatorial que envolve orientações voltadas para vários objetivos, seu tratamento poderá requerer o apoio de outros profissionais de saúde, além do médico. Sendo assim, a formação de uma equipe multiprofissional poderá propiciar essa ação diferenciada. $O$ trabalho em equipe multiprofissional poderá fornecer aos pacientes e à comunidade motivação para vencer o desafios e adotar atitudes que tornem as ações efetivas e permanentes, sendo assim poderá ser trabalhada maior quantidade de indivíduos que, com as diferentes abordagens, a adesão ao tratamento poderá ser alta e o número de pacientes com pressão arterial controlada e hábitos de vida saudáveis mais positivos, tendo cada paciente um replicador de conhecimentos sobre tais hábitos. 


\section{REFERÊNCIAS}

ARAÚJO, G. B. S.; GARCIA, T. R. Adesão ao tratamento anti-hipertensivo: uma análise conceitual. Revista Eletrônica de Enfermagem, v. 08, n. 02, p. 259 - 272; 2006.

BECKTE, N. S.; FLETCHER, A. E.; BULPITT, C. J. PROGRESS Collaborative Group. Randomised trial of a perindopril based blood pressure lowering regimen among 6105 individuals with previous stroke or transient ischaemic attack. Lancet. 358: 1033-41, 2001.

BIONDO-SIMOES, PESSOLE, M. L; et al. Wound healing: comparative study in hypertensive rats untreated and treated with an angiotensin converting enzime inhibitor. Rev. Col. Bras. Cir. Rio de Janeiro, v. 33, n. 2, 2006.

BERTOLDI, A. D; BARROS, A. J. D; HALLAL, P. C; et al. Utilização de medicamentos em adultos: prevalência e determinantes individuais. Rev. Saúde Pública. vol.38, no. 2, p.228238. ISSN 0034-8910, abr. 2004.

Camarano, A. A; Beltrão, K. I; PAscom, A. R. P.; MedeiRos, M. Goldani, A. M. Como vive o idoso? In: Camarano AA, organizador. Muito além dos 60: os novos idosos brasileiros. Rio de Janeiro: Instituto de Pesquisa Econômica Aplicada; p.19-71, 1999.

I Diretriz do Grupo de Estudos em Cardiogeriatria da Sociedade Brasileira de Cardiologia, Arquivos Brasileiros de Cardiologia. 79. supl I: $24-33,2002$.

IV Diretrizes Brasileiras de Hipertensão Arterial. Sociedade Brasileira de Hipertensão. Capítulo 2: Diagnóstico, Classificação, 2002.

V Diretrizes Brasileiras de Hipertensão Arterial. Sociedade Brasileira de Hipertensão. 2006. JACOMELLI, M.; SOUZA, R.; PEDREIRA J.; LEITE, W. Chronic cough in non-smokers. J. Pneumologia. São Paulo, v. 29, n. 6, 2003.

LEBRAO, M. L.; LAURENTI, R. Health, well-being and aging: the SABE study in São Paulo, Brazil. Rev. Bras. Epidemiol. São Paulo, v. 8, n. 2, 2005.

LORGA, A. et al. I Diretrizes do Grupo de Estudos em Cardiogeriatria da Sociedade Brasileira de Cardiologia. Arq. Bras. Cardiol. São Paulo, 2002.

SILVA, P. Anti-hipertensivos In: MAGALHÃES, L. B. N. C. Farmacologia. 7. ed. Rio de Janeiro: Guanabara Kogan, p. 700, 2006.

MIYASAKA, L.S.; ATALLAH, A. N. - Risk of drug Interactions: Combination of Antidepressants and other Drug. Rev. Saúde Pública. 37: 212-5, 2003.

NAKAO, N, YOSHIMURA, A.; MORITA, H.; TAKADA, M.; KAYANO, T.; IDEURA, T. Combination treatment of angiotensin II receptor blocker and angiotensin converting enzyme inhibitor in non-diabetic renal disease (COOPERATE): a randomized controlled trial. Lancet. 361:117-24, 2003. 
PRYDE, P.G.; THORPE, S. S.; LAMONT, C.A. Angiotensin-converting enzyme inhibitor fetopathy. J Am. Soc. Nphrol. 3: 1575-82,1993.

RAMOS, L.R. Os fármacos e o idoso in Terapêutica clínica no idoso. In: Gorzoni , Toniolo Neto. São Paulo: Ed. Sarvier, 3-7, 1995.

SACCO, M.; PELLEGRINI, F.; RONCAGLIONI, M.C.; AVANZINI, F.; TOGNONI, G.; NICOLUCCI, A. Primary prevention of cardiovascular events with low-dose aspirin and vitamin $E$ in type 2 diabetic patients: results of the Primary Prevention Project (PPP) trial. Diabetes Care, 26:326472, 2003.

SANS, S.; PALUZIE, G.; PUING, T.; BALAÑÁ, L.; BALAGUER, V. Prevalencia del consumo de medicamentos en lapoblación adulta de Cataluña. Gac Sanit, 16:121-30, 2002.

SANTELLO, J. L.; MION, J. D. Association of captopril and hydrochlorothiazide in mild and moderate hypertension. Arq. Bras. Cardiol, São Paulo, v. 71, n. 5, 1998.

SIMÕES, M.J.S.; FARACHE, F. A. Consumo de medicamentos em região do Estado de São Paulo (Brasil), 1985. Rev. Saúde Pública. 22 (6): 494-9, 1988.

SIMONETTI, J. P.; BATISTA, L.; CARVALHO, L. R. Habitos de salud y factores de riesgo en pacientes con hipertensión arterial. Rev. Latino-Am. Enfermagem. Ribeirão Preto, v. 10, n. 3, 2002.

SUCAR, D. D. Interação medicamentosa de venlafaxina com captopril. Rev. Bras. Psiquiatr. vol. 22, no. 3, p.134-137. ISSN 1516-4446, set 2000.

The Updated WHO/ISH Hypertension Guidelines 2004. Meeting of the International Society of Hypertension (ISH), São Paulo, Brazil 2004. http://www.medscape.com/viewarticle/ 471863. Acesso em 27 de Março 2007. 